\title{
THE SOLAR NEIGHBORHOOD. XXXIV. A SEARCH FOR PLANETS ORBITING NEARBY M DWARFS USING ASTROMETRY
}

\author{
John C. Lurie ${ }^{1,7}$, Todd J. Henry ${ }^{2,7}$, Wei-Chun Jao ${ }^{3,7}$, SAmuel N. Quinn ${ }^{3}$, Jennifer G. Winters ${ }^{3,7}$, \\ Philip A. Ianna ${ }^{2,7}$, David W. Koerner ${ }^{4}$, Adric R. Riedel ${ }^{5,7}$, and John P. Subasavage ${ }^{6,7}$ \\ ${ }^{1}$ Department of Astronomy, University of Washington, Seattle, WA 98195, USA; lurie@uw.edu \\ ${ }^{2}$ RECONS Institute, Chambersburg, PA 17201, USA \\ ${ }^{3}$ Department of Physics and Astronomy, Georgia State University, Atlanta, GA 30302, USA \\ ${ }_{5}^{4}$ Department of Physics and Astronomy, Northern Arizona University, Flagstaff, AZ 86011, USA \\ 5 Department of Astrophysics, American Museum of Natural History, New York, NY 10034, USA \\ ${ }^{6}$ United States Naval Observatory, Flagstaff, AZ 86001, USA \\ Received 2014 March 26; accepted 2014 July 16; published 2014 October 13
}

\begin{abstract}
Astrometric measurements are presented for seven nearby stars with previously detected planets: six M dwarfs (GJ 317, GJ 667C, GJ 581, GJ 849, GJ 876, and GJ 1214) and one K dwarf (BD-10 -3166). Measurements are also presented for six additional nearby $\mathrm{M}$ dwarfs without known planets, but which are more favorable to astrometric detections of low mass companions, as well as three binary systems for which we provide astrometric orbit solutions. Observations have baselines of 3 to 13 years, and were made as part of the RECONS long-term astrometry and photometry program at the CTIO/SMARTS $0.9 \mathrm{~m}$ telescope. We provide trigonometric parallaxes and proper motions for all 16 systems, and perform an extensive analysis of the astrometric residuals to determine the minimum detectable companion mass for the $12 \mathrm{M}$ dwarfs not having close stellar secondaries. For the six $\mathrm{M}$ dwarfs with known planets, we are not sensitive to planets, but can rule out the presence of all but the least massive brown dwarfs at periods of 2-12 years. For the six more astrometrically favorable $\mathrm{M}$ dwarfs, we conclude that none have brown dwarf companions, and are sensitive to companions with masses as low as $1 M_{\text {Jup }}$ for periods longer than two years. In particular, we conclude that Proxima Centauri has no Jovian companions at orbital periods of 2-12 years. These results complement previously published $\mathrm{M}$ dwarf planet occurrence rates by providing astrometrically determined upper mass limits on potential super-Jupiter companions at orbits of two years and longer. As part of a continuing survey, these results are consistent with the paucity of super-Jupiter and brown dwarf companions we find among the over 250 red dwarfs within 25 pc observed longer than five years in our astrometric program.
\end{abstract}

Key words: astrometry - planetary systems - solar neighborhood - stars: low-mass

\section{INTRODUCTION}

During the brief history of extrasolar planet investigations, our understanding of the relative populations of different types of planets has been limited by the observational biases of the techniques employed. With the advent of sophisticated transit searches and hypersensitive radial velocity measurements, significant progress has been made discovering various types of planets that orbit stars with periods up to a few years. Less progress has been made in discovering planets in longer orbits, and particularly around nearby $\mathrm{M}$ dwarfs, which account for at least $74 \%$ of the stellar population within $10 \mathrm{pc}$ (Henry et al. 2006). M dwarfs offer fertile ground for companion searches, as Dressing \& Charbonneau (2013) have inferred that a high fraction of $\mathrm{M}$ dwarfs host terrestrial planets at short orbital periods. Less is known about the populations of Jupitermass planets and brown dwarfs around $\mathrm{M}$ dwarfs, particularly at orbital periods longer than a few years.

To understand how $\mathrm{M}$ dwarf planetary systems form and evolve, we must probe the full regime of companion masses and orbital periods. Transit techniques are geometrically biased toward companions with small orbits, while radial velocity techniques are biased toward massive companions with short periods that exert large gravitational accelerations on their host

\footnotetext{
7 Visiting Astronomer, Cerro Tololo Inter-American Observatory. CTIO is operated by the Association of Universities for Research in Astronomy, Inc., under contract to the National Science Foundation.
}

stars. Direct imaging techniques are limited to young, giant planets at large separations. Astrometric techniques, which measure the positions of stars on the plane of the sky, are most sensitive to Jovian-type planets in Jovian-type orbits. While radial velocity observing programs are now becoming sensitive to such companions (Bonfils et al. 2013a; Montet et al. 2014), the astrometric results presented here have longer observational baselines, of up to 13 years. Furthermore, astrometry can detect companions with a large range of inclinations and orientations, and allow for the determination of orbit inclinations and accurate companion masses.

To date the majority of nearby extrasolar planets around $\mathrm{M}$ dwarfs have been discovered by radial velocity searches, which tend to select the brightest $\mathrm{M}$ dwarfs. As discussed in more detail in Section 3, in ground-based imaging programs the brightest targets generally have the noisiest astrometric residuals due to the short exposures required and the lack of comparably bright reference stars. With the exception of GJ 1214 , five M dwarfs in our sample were found to have planets using radial velocity techniques, and are among the brightest targets in our astrometric program. An extreme case is the $\mathrm{K}$ dwarf BD-10 3166, for which we are not sensitive to sub-stellar companions, but for which we provide the first accurate parallax. For comparison, we have included six additional M dwarfs that are less bright, less massive, and closer, and therefore more favorable to companion detection via astrometry. To calibrate our analysis, we have also included three 
confirmed stellar binaries with clear photocentric perturbations for which we have characterized the orbits. These binaries were chosen from the roughly two dozen binaries in our observing program with clear astrometric perturbations because we have observed multiple orbital periods, and can most accurately characterize the orbits.

Astrometric solutions for proper motion and parallax are given for each of the 16 systems targeted, plus orbital solutions for three binaries. A detailed analysis of the astrometric residuals is given to search for companions to the $12 \mathrm{M}$ dwarf systems without close stellar companions. Periodograms of the astrometric residuals have been generated, along with detection limits based on simulations of 10 million hypothetical companions to each star. These are the first results of a larger RECONS $^{8}$ survey for companions orbiting more than 250 red dwarfs within $25 \mathrm{pc}$ for which we have at least five years of coverage. As observations continue, this sample will grow, further constraining the population of brown dwarf and superJupiter companions in long period orbits around $\mathrm{M}$ dwarfs. Finally, to provide context for these results we provide a comprehensive list of the $17 \mathrm{M}$ dwarfs within $25 \mathrm{pc}$ having exoplanets as of 2014 July 1, including the six targeted in this work.

\section{OBSERVATIONS AND REDUCTIONS}

\subsection{Astrometry}

The $0.9 \mathrm{~m}$ telescope at CTIO is equipped with a $2048 \times 2048$ Tektronix CCD camera with $0^{\prime \prime} 401$ pixel $^{-1}$ plate scale (Jao et al. 2003). Only the center quarter of the chip is used for astrometric and photometric observations, yielding a $6^{\prime} .8$ square field of view. Astrometric exposures are taken through one of four filters, $V_{\mathrm{J}}$ (old), $V_{\mathrm{J}}$ (new), $R_{\mathrm{KC}}$, or $I_{\mathrm{KC}}{ }^{9}$ (hereafter without subscripts, and the $V$ filters combined). Depending on the brightnesses of the science targets, reference stars, and sky conditions, exposure times vary from 20 to $1200 \mathrm{~s}$ for targets with $9 \leqslant V R I \leqslant 19$. For optimal centroiding, exposure times are set so that either science or reference stars have maximum peak ADU of $\sim 50,000$ (digital saturation occurs at 65,537 ADU). Observations are almost always made within \pm 30 minutes of a science target's transit to minimize the corrections required for differential color refraction, as described in Jao et al. (2005). Three to five frames are typically taken each night, depending primarily on the exposure time required. To enable routine calibration of the science images, bias and dome flat frames are taken nightly.

Instrument setups for most stars have been kept constant during the 13 years of observations. However, we have used two $V$ filters, dubbed the "old" Tek\#2 $V$ filter $\left(\lambda_{\text {central }}\right.$ $=5438 \AA, \Delta \lambda=1026 \AA)$ and "new" Tek\#1 V filter $\left(\lambda_{\text {central }}\right.$ $=5475 \AA, \Delta \lambda=1000 \AA)$, because the "old" filter cracked in 2005 February. The "new" $V$ filter was used between 2005 and 2009. The "old" $V$ filter was reinstated in 2009 July after confirming that the crack in the corner did not significantly affect astrometric residuals. As discussed in Subasavage et al. (2009), a reliable parallax can be obtained using data from both filters as long as at least 1-2 years of data (depending on observing frequency) have been taken in each filter. Reductions containing both "old" and "new" $V$ frames can exhibit offsets

\footnotetext{
8 REsearch Consortium on Nearby Stars, www.recons.org

9 The central wavelengths for the $V_{\mathrm{J}}$ (old), $V_{\mathrm{J}}$ (new), $R_{\mathrm{KC}}$, and $I_{\mathrm{KC}}$ filters are 5438, 5475, 6425, and $8075 \AA$, respectively.
}

of a several milliarcseconds (mas) in residuals on both axes. This has been mitigated by choosing close-in reference stars, and only using frames taken near the meridian. In total, 7 of the 16 systems discussed in this paper were observed astrometrically in the $V$ filter. Further details about the filters and their effects on the astrometry can be found in Subasavage et al. (2009) and Riedel et al. (2010).

The paths traced on the sky by science stars result from the combinations of proper motions and parallactic shifts. Details of the data reduction process used to separate these motions are given in Jao et al. (2005) and Henry et al. (2006). Briefly, we (1) use SExtractor (Bertin \& Arnouts 1996) to measure centroids, (2) define a six-constant plate model to find plate constants (given in Equation (4) of Jao et al. 2005), (3) assume that ensembles of reference stars have zero mean parallax and proper motion, (4) solve least-square equations for multi-epoch images (given in Equation (5) of Jao et al. 2005), and (5) convert from relative parallax to absolute parallax by estimating the distances of the reference stars photometrically. Our typical centering precision is $2.1-3.5$ mas, or $0.5 \%-0.9 \%$ of a pixel, depending on the filter, with $I$ being the best and $V$ being the worst. To correct the relative parallax to an absolute parallax, photometric distances are estimated by comparing $V R I$ colors to $M_{V}$ for single, main-sequence stars in the RECONS 10 pc sample (Henry et al. 1997, 2006). A distance is estimated for each reference star, and the correction to absolute parallax is then computed using the weighted mean distance of the entire reference field. The uncertainty on the correction is determined using Equation (6) in Jao et al. (2005).

In the case of a binary with a given combination of magnitude and mass differences, ${ }^{10}$ we detect its photocenter orbit around its barycenter, in addition to the motions due to parallax and proper motion. Hence, the residuals of our typical binary star's parallax reduction are significantly offset from zero. In order to get a better parallax result and calculate the photocenter's orbital elements, we first carry out a standard reduction for proper motion and parallax. We then fit a photocentric orbit to the residuals, i.e, we treat these residuals as a binary orbit, using the techniques described in Hartkopf et al. (1989). Based on the orbital elements we calculate, this photocentric orbit in components of right ascension (R.A.) and declination (decl.) is then removed from the centroids of the science star at each epoch. Finally, we re-calculate proper motion and parallax using these corrected centroids. The final "cleaned" proper motions and parallaxes are the values given for the binaries in Table 1. After one iteration, the parallax errors are reduced to those typical of similar program stars, and the residuals are significantly reduced and consistent with the mean errors found for our overall program.

\subsection{Photometry}

VRI photometry was obtained at the CTIO $0.9 \mathrm{~m}$ using the same instrumental setup used for the astrometry frames. As for astrometry observations, bias and dome flat frames are taken nightly for basic image calibration. All science stars were observed at airmass $<1.8$. Exposure times were chosen to reach

\footnotetext{
$\overline{10}$ As described in van de $\operatorname{Kamp}(1967), \alpha=(B-\beta) a$, where $\alpha$ is the photocentric semimajor axis of the orbit of the primary, $B$ is the fractional mass $\left(M_{B} /\left(M_{A}+M_{B}\right)\right), \beta$ is the relative luminosity $\left(1 /\left(1+10^{(0.4) \Delta m}\right)\right)$, and $a$ is the semimajor axis of the relative orbit of the two components. The perturbation we detect here is $\alpha$.
} 
Table 1

Astrometric Results

\begin{tabular}{|c|c|c|c|c|c|c|c|c|c|c|c|c|c|c|c|c|c|}
\hline $\begin{array}{l}\text { Name } \\
(1)\end{array}$ & $\begin{array}{c}\text { R.A. } \\
\text { (J2000.0) } \\
(2)\end{array}$ & $\begin{array}{c}\text { Decl. } \\
(\mathrm{J} 2000.0) \\
(3)\end{array}$ & $\begin{array}{l}\text { Fil. } \\
\text { (4) }\end{array}$ & $\begin{array}{c}\mathrm{N}_{\text {sea }} \\
(5)\end{array}$ & $\begin{array}{l}N_{\text {frm }} \\
(6)\end{array}$ & $\begin{array}{c}\text { Coverage } \\
\text { (7) }\end{array}$ & $\begin{array}{c}\text { Years } \\
(8)\end{array}$ & $\begin{array}{c}N_{\text {ref }} \\
(9)\end{array}$ & $\begin{array}{c}\pi(\mathrm{rel}) \\
(\mathrm{mas}) \\
(10)\end{array}$ & $\begin{array}{c}\pi \\
\text { (corr) } \\
(\mathrm{mas}) \\
(11)\end{array}$ & $\begin{array}{c}\pi(\mathrm{abs}) \\
(\mathrm{mas}) \\
(12)\end{array}$ & $\begin{array}{c}\mu \\
\left(\operatorname{mas~yr}^{-1}\right) \\
(13)\end{array}$ & $\begin{array}{l}\text { P.A. } \\
(\mathrm{deg}) \\
(14)\end{array}$ & $\begin{array}{c}V_{\tan } \\
\left(\mathrm{km} \mathrm{s}^{-1}\right) \\
(15)\end{array}$ & $\begin{array}{l}\text { Obs. } \\
\text { Prec. } \\
\text { (mas) } \\
(16)\end{array}$ & $\begin{array}{c}\text { Perc. } \\
\text { Cmpn. } \\
(17)\end{array}$ & $\begin{array}{c}\text { Notes } \\
(18)\end{array}$ \\
\hline \multicolumn{18}{|c|}{ Extrasolar Planet Hosts } \\
\hline GJ 317 & 084059.21 & -232722.6 & $\mathrm{R}$ & $5 c$ & 75 & 2009.04-2013.38 & 4.35 & 7 & $\begin{array}{c}64.04 \\
\pm 1.45\end{array}$ & $\begin{array}{c}1.50 \\
\pm 0.50\end{array}$ & $\begin{array}{c}65.54 \\
\pm 1.53\end{array}$ & $\begin{array}{l}930.7 \\
\pm 1.1\end{array}$ & $\begin{array}{c}330.5 \\
\pm 0.13\end{array}$ & 65.5 & 4.99 & & \\
\hline BD-10 3166 & 105828.79 & -104613.4 & I & $7 \mathrm{~s}$ & 71 & $2004.43-2011.50$ & 7.07 & 6 & $\begin{array}{c}13.84 \\
\pm 3.04\end{array}$ & $\begin{array}{l}1.50 \\
\pm 0.50\end{array}$ & $\begin{array}{c}15.34 \\
\pm 3.08\end{array}$ & $\begin{array}{l}185.9 \\
\pm 1.5\end{array}$ & $\begin{array}{c}269.1 \\
\pm 0.67\end{array}$ & 52.4 & 10.85 & & \\
\hline GJ 581 & 151926.83 & -074320.1 & V & $14 \mathrm{~s}$ & 267 & 2000.58-2013.38 & 12.80 & 11 & $\begin{array}{l}157.67 \\
\pm 1.57\end{array}$ & $\begin{array}{c}1.12 \\
\pm 0.17\end{array}$ & $\begin{array}{l}158.79 \\
\pm 1.58\end{array}$ & $\begin{array}{c}1224.3 \\
\pm 0.4\end{array}$ & $\begin{array}{c}266.0 \\
\pm 0.03\end{array}$ & 36.5 & 7.93 & $96 \%$ & $\mathrm{a}$ \\
\hline GJ 1214 & 171518.92 & +04 5750.1 & I & $4 c$ & 80 & 2010.39-2013.38 & 3.00 & 9 & $\begin{array}{c}68.20 \\
\pm 1.26\end{array}$ & $\begin{aligned} & 1.88 \\
\pm & 0.18\end{aligned}$ & $\begin{array}{l}70.08 \\
\pm 1.27\end{array}$ & $\begin{array}{l}945.5 \\
\pm 1.4\end{array}$ & $\begin{array}{c}142.0 \\
\pm 0.17\end{array}$ & 63.9 & 5.02 & & \\
\hline GJ 667C & 171858.82 & -345948.6 & $\mathrm{~V}$ & $11 \mathrm{~s}$ & 140 & 2003.52-2013.38 & 9.86 & 5 & $\begin{array}{l}139.38 \\
\pm 1.98\end{array}$ & $\begin{array}{c}1.50 \\
\pm 0.50\end{array}$ & $\begin{array}{l}140.88 \\
\pm 2.04\end{array}$ & $\begin{array}{c}1154.1 \\
\pm 0.6\end{array}$ & $\begin{array}{c}101.0 \\
\pm 0.05\end{array}$ & 38.8 & 7.66 & $93 \%$ & \\
\hline GJ 849 & 220940.34 & -043826.8 & $\mathrm{~V}$ & $11 \mathrm{~s}$ & 135 & 2003.52-2013.39 & 9.86 & 5 & $\begin{array}{l}113.78 \\
\pm 1.97\end{array}$ & $\begin{array}{c}2.27 \\
\pm 0.30\end{array}$ & $\begin{array}{l}116.05 \\
\pm 1.99\end{array}$ & $\begin{array}{c}1118.0 \\
\pm 0.5\end{array}$ & $\begin{array}{c}90.8 \\
\pm 0.04\end{array}$ & 45.7 & 9.53 & $81 \%$ & \\
\hline GJ 876 & 225316.75 & -141549.2 & $\mathrm{~V}$ & $11 \mathrm{~s}$ & 85 & 2003.52-2013.39 & 9.87 & 6 & $\begin{array}{l}210.97 \\
\pm 3.99\end{array}$ & $\begin{array}{c}2.14 \\
\pm 0.57\end{array}$ & $\begin{array}{l}213.11 \\
\pm 4.03\end{array}$ & $\begin{array}{c}1149.4 \\
\pm 1.1\end{array}$ & $\begin{array}{c}125.7 \\
\pm 0.11\end{array}$ & 25.6 & 8.43 & $99 \%$ & \\
\hline \multicolumn{18}{|c|}{ Best Case Targets } \\
\hline GJ 1061 & 033559.72 & -443045.5 & $\mathrm{R}$ & $13 \mathrm{~s}$ & 194 & 1999.62-2012.95 & 13.32 & 7 & $\begin{array}{l}269.92 \\
\pm 1.29\end{array}$ & $\begin{array}{c}0.94 \\
\pm 0.08\end{array}$ & $\begin{array}{l}270.86 \\
\pm 1.29\end{array}$ & $\begin{array}{l}827.7 \\
\pm 0.3\end{array}$ & $\begin{array}{c}117.7 \\
\pm 0.04\end{array}$ & 14.5 & 7.59 & $79 \%$ & a \\
\hline LP 944-020 & 033935.25 & -352543.8 & I & $8 \mathrm{~s}$ & 59 & 2003.95-2012.94 & 8.99 & 10 & $\begin{array}{l}154.53 \\
\pm 1.03\end{array}$ & $\begin{array}{c}1.36 \\
\pm 0.10\end{array}$ & $\begin{array}{l}155.89 \\
\pm 1.03\end{array}$ & $\begin{array}{l}408.3 \\
\pm 0.3\end{array}$ & $\begin{array}{c}48.5 \\
\pm 0.07\end{array}$ & 12.4 & 2.13 & $94 \%$ & a \\
\hline GJ 1128 & 094246.36 & -685306.1 & $\mathrm{~V}$ & $13 \mathrm{~s}$ & 167 & 2000.23-2013.12 & 12.89 & 8 & $\begin{array}{l}153.54 \\
\pm 0.75\end{array}$ & $\begin{array}{c}0.73 \\
\pm 0.11\end{array}$ & $\begin{array}{l}154.27 \\
\pm 0.76\end{array}$ & $\begin{array}{c}1123.0 \\
\pm 0.2\end{array}$ & $\begin{array}{c}356.1 \\
\pm 0.02\end{array}$ & 34.5 & 3.17 & $80 \%$ & a \\
\hline DENIS J1048-3956 & 104814.56 & -395607.0 & I & $13 \mathrm{~s}$ & 200 & 2001.15-2013.27 & 12.13 & 11 & $\begin{array}{l}247.23 \\
\pm 0.60\end{array}$ & $\begin{array}{c}0.85 \\
\pm 0.10\end{array}$ & $\begin{array}{l}248.08 \\
\pm 0.61\end{array}$ & $\begin{array}{l}1531.6 \\
\pm 0.2\end{array}$ & $\begin{array}{l}229.5 \\
\pm 0.01\end{array}$ & 29.3 & 2.92 & $97 \%$ & a \\
\hline SCR 1138-7721 & 113816.76 & -772148.5 & $\mathrm{I}$ & $11 \mathrm{~s}$ & 134 & $2003.25-2013.27$ & 10.03 & 12 & $\begin{array}{l}119.60 \\
\pm 1.01\end{array}$ & $\begin{array}{c}0.81 \\
\pm 0.07\end{array}$ & $\begin{array}{l}120.41 \\
\pm 1.01\end{array}$ & $\begin{array}{c}2143.3 \pm \\
0.4\end{array}$ & $\begin{array}{c}287.8 \pm \\
0.02\end{array}$ & 84.4 & 4.20 & $69 \%$ & a \\
\hline Proxima Cen & 142943.02 & -624046.7 & $\mathrm{~V}$ & $14 \mathrm{~s}$ & 205 & $2000.57-2013.25$ & 12.68 & 5 & $\begin{array}{c}766.41 \pm \\
0.91\end{array}$ & $\begin{array}{c}1.72 \pm \\
0.50\end{array}$ & $\begin{array}{c}768.13 \pm \\
1.04\end{array}$ & $\begin{array}{c}3850.8 \pm \\
0.6\end{array}$ & $\begin{array}{c}282.4 \pm \\
0.02\end{array}$ & 23.8 & 4.83 & $99 \%$ & a \\
\hline \multicolumn{18}{|c|}{ Confirmed Binaries } \\
\hline LHS $1582 \mathrm{AB}$ & 034322.08 & -093350.9 & $\mathrm{R}$ & $11 \mathrm{~s}$ & 102 & 2000.87-2012.94 & 12.06 & 7 & $\begin{array}{c}48.84 \pm \\
1.18\end{array}$ & $\begin{array}{c}2.00 \pm \\
0.26\end{array}$ & $\begin{array}{c}50.84 \pm \\
1.21\end{array}$ & $\begin{array}{c}509.4 \pm \\
0.3\end{array}$ & $\begin{array}{c}52.7 \pm \\
0.06\end{array}$ & 47.5 & 3.88 & & $\mathrm{a}$ \\
\hline GJ 748AB & 191214.60 & +025311.0 & $\mathrm{V}$ & $10 \mathrm{~s}$ & 154 & 2004.45-2013.39 & 8.95 & 11 & $\begin{array}{c}97.77 \pm \\
1.15\end{array}$ & $\begin{array}{c}2.22 \pm \\
0.41\end{array}$ & $\begin{array}{c}99.99 \pm \\
1.22\end{array}$ & $\begin{array}{c}1857.8 \pm \\
0.5\end{array}$ & $\begin{array}{c}107.4 \pm \\
0.02\end{array}$ & 88.1 & 5.80 & & \\
\hline LHS 3738AB & 215849.13 & -322625.5 & $\mathrm{R}$ & $12 \mathrm{~s}$ & 151 & 1999.64-2012.81 & 13.17 & 12 & $\begin{array}{c}50.82 \pm \\
1.01\end{array}$ & $\begin{array}{c}1.40 \pm \\
0.21\end{array}$ & $\begin{array}{c}52.22 \pm \\
1.03\end{array}$ & $\begin{array}{c}535.2 \pm \\
0.3\end{array}$ & $\begin{array}{c}229.1 \pm \\
0.06\end{array}$ & 48.6 & 2.50 & & $\mathrm{a}$ \\
\hline
\end{tabular}

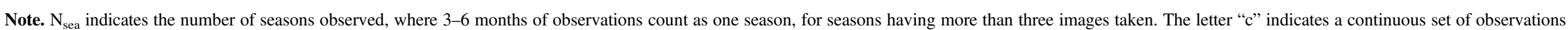

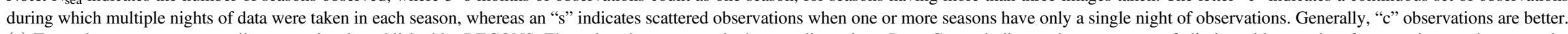

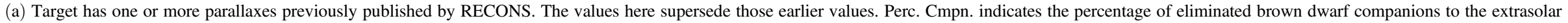
planet hosts, and the percentage of eliminated planetary-mass companions to the best case targets. 
a signal-to-noise ratio $(\mathrm{S} / \mathrm{N})>100$ for science stars in each of the VRI filters. Combinations of fields that provided 10 or more standard stars from Landolt $(1992,2007)$ and/or E-regions from Graham (1982) were observed several times each night to derive transformation equations and extinction curves. Further details of photometric data reductions, the definition of transformation equations, errors, etc., can be found in Jao et al. (2005) and Winters et al. (2011).

\subsection{Spectroscopy}

Spectroscopic observations used to the provide spectral types in Table 2 were made at the CTIO $1.5 \mathrm{~m}$ using the R-C spectrograph and Loral $1200 \times 800$ CCD camera between 2003 and 2006. Grating \#32 was used in first order with a tilt of $15^{\circ}$. 1 , and observations were made using a $2^{\prime \prime}$ slit. The order-blocking filter OG570 was utilized to provide spectra covering the range of $6000-9000 \AA$ with a resolution of $8.6 \AA$. For calibration, bias frames, dome flats, and sky flats were taken at the beginning of each night. Further details regarding the $1.5 \mathrm{~m}$ spectroscopy program and associated data reduction, including assignment of spectral types, can be found in Henry et al. (2004).

\section{ANALYSIS}

Astrometric residuals represent the deviation in a target's measured position from the solution for proper motion and parallax, given for all 16 target stars in Table 1. The residuals for each star are plotted in Figure 1. Each filled circle represents the mean of typically three to five frames taken in a single night, with a corresponding estimate of the nightly mean error. Open circles represent nights with only one frame, which are included in the parallax measurement but are not included in the following analysis of the residuals. The three binaries (GJ $748 \mathrm{AB}$, LHS 1582AB, and LHS 3738AB) exhibit large, periodic perturbations in both R.A. and decl., indicating the presence of a companion in each case. Because the systems are unresolved in our images, we calculate a photocentric semimajor axis, as previously discussed. The remaining targets generally have flat residuals, although some are more scattered (GJ 849) than others (GJ 1128). Targets with the flattest residuals, such as DENIS J1048-3956, generally have at least 8 reference stars that closely and evenly surround the target, and are bright enough to have a least 1,000 peak counts when the target has 50,000. Fainter targets tend to have the flattest residuals because they have a larger number of suitable reference stars and require longer exposures, which smooth out short-term seeing effects that produce PSFs with poorlydefined centroids.

The residuals were analyzed for the presence of companions using Lomb-Scargle periodograms, shown in Figure 2, generated by the method given in Zechmeister \& Kürster (2009). This method weights each data point by its estimated nightly mean error, and produces a normalized periodogram for the data sequence with power ranging from 0 and 1 . We only attempt to detect companions with periods greater than two years but less than the length of the observations, and have only generated periodograms for those ranges. The data were oversampled in increments of one day to create smooth periodograms. As Frescura et al. (2008) have shown, oversampling the periodogram does not significantly increase the risk of false periodogram peaks.
As the data are irregularly spaced, it was not possible to use an analytical formula for the false alarm probabilities (FAPs). Instead, empirical FAP distribution functions were calculated as outlined in Frescura et al. (2008) by generating periodograms for 10,000 sets of random noise. Each of the 10,000 data sets has the same number of observations as the actual data, with the same observation times and errors as the actual data. The randomized values of each point within a given data set are assigned from a normal distribution with a standard deviation representing the distribution of offsets from zero of the actual data points.

Each of these 10,000 periodograms was then sampled in a grid of periods with five day increments between two years and the length of observations to find the highest power occurring in each periodogram. The FAP for an observed periodogram peak of a given power is equal to the fraction of the 10,000 data sets with peaks greater than or equal to that power. The normalized powers corresponding to FAP values of $0.1,0.01$, and 0.001 are shown as dashed lines in Figure 2. This corresponds to $10 \%, 1 \%$, and $0.1 \%$ probabilities that the peak is due solely to random noise and the cadence of observations. As a calibration, periodograms and FAP functions were also generated for the three confirmed stellar binaries for which astrometric perturbations are clearly evident. These binaries have significant periodogram peaks $(\mathrm{FAP}<0.001)$ in both the R.A. and decl. axes at periods corresponding to their orbital periods, while the remaining 13 targets have no significant peaks. This indicates that our periodograms are sensitive to astrometric perturbations, and that we have not detected any significant periodicity in the residuals of the remaining $12 \mathrm{M}$ dwarfs and one $\mathrm{K}$ dwarf without companions.

Given that there are no companions evident in the residuals of the non-binary targets, we aim to establish lower limits for the companion masses and periods to which we are sensitive, and would have detected were they present. For each target we ran a simulation of 10 million hypothetical companions orbiting each star with masses chosen randomly from a uniform distribution between 0.5 and $80 M_{\text {Jup }}$, and periods chosen randomly from a uniform distribution between 2 years up to the length of observations. Although we have observed perturbations as short as 1.2 years, our practical lower limit is 2 years. All geometric parameters (inclination, eccentricity, time of periastron, longitude of ascending node, and longitude of periastron) were assigned randomly from a uniform distribution of all possible values, including eccentricity, which was allowed to be as high as 0.99 .

The program then calculates the astrometric perturbation that each simulated companion would induce on the primary, using a primary mass calculated from the mass-luminosity relations in Henry \& McCarthy (1993) (Equation $(5 a, b)$ ) and the revised relation for the lowest mass stars in Henry et al. (1999). For DEN J1048-3956 and LP 944-020, which are too faint for the relations, we assume masses of $0.08 M_{\odot}$. We assume that the companion does not contribute significantly to the overall flux in each system, so that the photocenter of the system is concentric with the primary star. This assumption proves problematic when considering brown dwarf companions to the two latest M dwarfs, DEN J1048-3956 and LP 944-020. However, the trigonometric parallaxes of these targets agree well with their photometric distance estimates (Columns 12 and 13 of Table 2), and we conclude that neither have nearly equal luminosity companions. 
Table 2

Photometric and Spectroscopic Results

\begin{tabular}{|c|c|c|c|c|c|c|c|c|c|c|c|c|c|c|}
\hline Name & $\begin{array}{c}V \\
(\mathrm{mag}) \\
(2)\end{array}$ & $\begin{array}{c}R \\
(\mathrm{mag}) \\
(3)\end{array}$ & $\begin{array}{c}I \\
(\mathrm{mag}) \\
(4)\end{array}$ & (5) & $\begin{array}{c}J \\
(\mathrm{mag}) \\
(6)\end{array}$ & $\begin{array}{c}H \\
(\mathrm{mag}) \\
(7)\end{array}$ & $\begin{array}{c}K_{S} \\
(\mathrm{mag}) \\
(8)\end{array}$ & $\begin{array}{l}\text { Spectral } \\
\text { type } \\
(9)\end{array}$ & (10) & $\begin{array}{c}\text { Mass } \\
\left(M_{\odot}\right) \\
(11)\end{array}$ & $\begin{array}{l}\text { Trig. dist. } \\
\text { (pc) } \\
(12)\end{array}$ & $\begin{array}{l}\text { Phot. dist. } \\
\qquad(\mathrm{pc}) \\
(13)\end{array}$ & $\begin{array}{c}\text { No. of } \\
\text { relations } \\
(14)\end{array}$ & $\begin{array}{l}\text { Notes } \\
(15) \\
\end{array}$ \\
\hline \multicolumn{15}{|c|}{ Extrasolar Planet Hosts } \\
\hline GJ 317 & 12.01 & 10.84 & 9.37 & 3 & 7.934 & 7.321 & 7.028 & M3.5 V & 1 & 0.35 & $15.26 \pm 0.36$ & $9.70 \pm 1.53$ & 12 & \\
\hline BD-10 3166 & 10.03 & 9.58 & 9.19 & 3 & 8.611 & 8.300 & 8.124 & $\mathrm{~K} 3.0 \mathrm{~V}$ & 2 & 0.85 & $65.19 \pm 13.64$ & & & a \\
\hline GJ 581 & 10.56 & 9.44 & 8.03 & 3 & 6.706 & 6.095 & 5.837 & M3.0 V & 2 & 0.30 & $6.30 \pm 0.06$ & $6.60 \pm 1.03$ & 12 & \\
\hline GJ 1214 & 14.71 & 13.27 & 11.50 & 3 & 9.750 & 9.094 & 8.782 & $\mathrm{M} 4.5 \mathrm{~V}$ & 1 & 0.14 & $14.27 \pm 0.24$ & $12.42 \pm 2.00$ & 12 & \\
\hline GJ $667 \mathrm{C}$ & 10.34 & 9.29 & 8.09 & 3 & 6.848 & 6.322 & 6.036 & M1.5 V & 2 & 0.36 & $7.10 \pm 0.10$ & $9.41 \pm 1.49$ & 12 & \\
\hline GJ 849 & 10.38 & 9.27 & 7.87 & 3 & 6.510 & 5.899 & 5.594 & $\mathrm{M} 3.0 \mathrm{~V}$ & 2 & 0.42 & $8.62 \pm 0.15$ & $5.73 \pm 0.92$ & 12 & \\
\hline GJ 876 & 10.18 & 8.97 & 7.40 & 3 & 5.934 & 5.349 & 5.010 & M3.5 V & 2 & 0.27 & $4.69 \pm 0.09$ & $3.46 \pm 0.54$ & 12 & \\
\hline \multicolumn{15}{|c|}{ Best Case Targets } \\
\hline GJ 1061 & 13.09 & 11.45 & 9.47 & 6 & 7.523 & 7.015 & 6.610 & $\mathrm{M} 5.0 \mathrm{~V}$ & 3 & 0.11 & $3.69 \pm 0.02$ & $3.55 \pm 0.60$ & 12 & \\
\hline LP 944-020 & 18.69 & 16.39 & 13.98 & 3 & 10.725 & 10.017 & 9.548 & M9.0 V & 4 & 0.08 & $6.42 \pm 0.04$ & $7.04 \pm 1.32$ & 11 & \\
\hline GJ 1128 & 12.74 & 11.36 & 9.62 & 3 & 7.953 & 7.385 & 7.037 & $\mathrm{M} 4.0 \mathrm{~V}$ & 2 & 0.15 & $6.48 \pm 0.03$ & $6.33 \pm 1.00$ & 12 & \\
\hline DENIS J1048-3956 & 17.37 & 14.98 & 12.47 & 4 & 9.538 & 8.905 & 8.447 & $\mathrm{M} 8.0 \mathrm{~V}$ & 2 & 0.08 & $4.03 \pm 0.01$ & $4.48 \pm 0.73$ & 10 & \\
\hline SCR 1138-7721 & 14.78 & 13.20 & 11.24 & 4 & 9.399 & 8.890 & 8.521 & $\mathrm{M} 5.0 \mathrm{~V}$ & 3 & 0.11 & $8.31 \pm 0.07$ & $9.45 \pm 1.71$ & 12 & \\
\hline Proxima Cen & 11.13 & 9.45 & 7.41 & 3 & 5.357 & 4.835 & 4.384 & M5.0 V & 2 & 0.11 & $1.30 \pm 0.01$ & $1.15 \pm 0.18$ & 12 & $\mathrm{~b}$ \\
\hline \multicolumn{15}{|c|}{ Confirmed Binaries } \\
\hline LHS $1582 \mathrm{AB}$ & $14.69 \mathrm{~J}$ & $13.33 \mathrm{~J}$ & $11.60 \mathrm{~J}$ & 4 & $9.799 \mathrm{~J}$ & $9.177 \mathrm{~J}$ & $8.854 \mathrm{~J}$ & $\mathrm{M} 4.5 \mathrm{VJ}$ & 1 & & $19.67 \pm 0.47$ & $13.27 \pm 2.25$ & 12 & $\mathrm{c}$ \\
\hline GJ 748AB & $11.10 \mathrm{~J}$ & $9.95 \mathrm{~J}$ & $8.47 \mathrm{~J}$ & 3 & $7.087 \mathrm{~J}$ & $6.572 \mathrm{~J}$ & $6.294 \mathrm{~J}$ & $\mathrm{M} 3.5 \mathrm{VJ}$ & 2 & & $10.00 \pm 0.12$ & $7.69 \pm 1.26$ & 12 & c \\
\hline LHS 3738AB & $15.78 \mathrm{~J}$ & $14.29 \mathrm{~J}$ & $12.46 \mathrm{~J}$ & 3 & $10.654 \mathrm{~J}$ & $10.091 \mathrm{~J}$ & $9.761 \mathrm{~J}$ & M4.5 VJ & 5 & & $19.15 \pm 0.38$ & $18.50 \pm 2.96$ & 12 & c \\
\hline
\end{tabular}

Notes: (a) Distance estimate only applicable to M dwarfs; (b) Actual error is $\pm 0.002 \mathrm{pc}$ in trigonometric distance; (c) "J" signifies joint photometry and spectroscopy for unresolved binaries.

References. For spectral types: (1) Reid et al. (1995); (2) This work; (3) Henry et al. (2006); (4) Dieterich et al. (2014); (5) Hawley et al. (1996) 

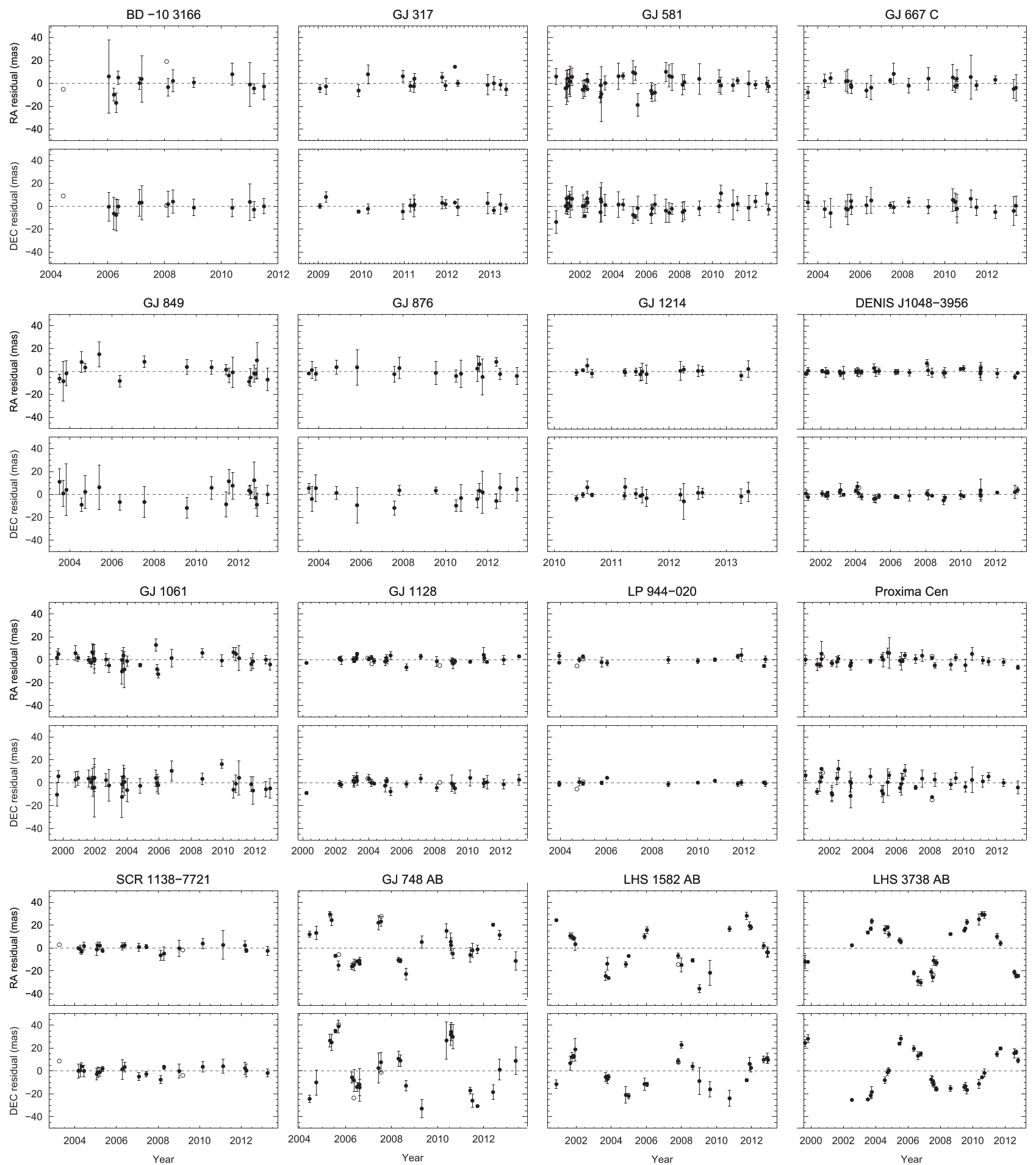

Figure 1. Astrometric residuals plotted in right ascension (R.A.) and declination (decl.), in units of milliarcseconds (mas). Filled circles represent the mean of typically three to five frames taken in a single night. Open circles represent nights for which there is only one frame. All panels are on a \pm 50 mas vertical scale.

The goodness-of-fit between the observed data $y_{i}$ with errors $\sigma_{i}$, and a flat line with $\tilde{y_{i}}=0$ was determined using the reduced chi squared statistic

$$
\chi_{\mathrm{red}}^{2}=\frac{\chi^{2}}{K}=\frac{1}{K} \sum_{i=1}^{N}\left(\frac{y_{i}-\tilde{y}_{i}}{\sigma_{i}}\right)^{2},
$$

where $K$ is the number of degrees of freedom, given by $N-P-1$ for $N$ data points and $P$ fitted parameters. Because we did not attempt to fit a model to the data, but only to analyze how well a simulation fits the data, we set $P$ equal to zero. As data points with small error bars are more heavily weighted, we discarded epochs that have unrealistic errors smaller than 1 mas -representing less than 5\% of the epochs-because such points overconstrain the orbits that can be fit.

Simulated orbits for which $\chi_{\text {red }}^{2}$ was greater than 4 in at least one of either R.A. or decl. we consider to be orbits that we would have detected. This threshold of 4 is based empirically 


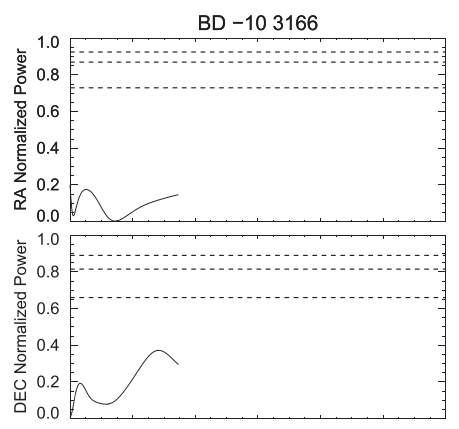

GJ 849
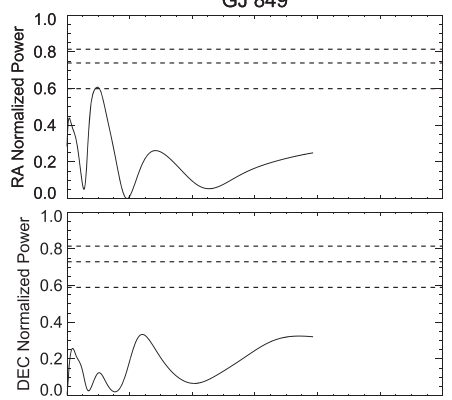

GJ 1061
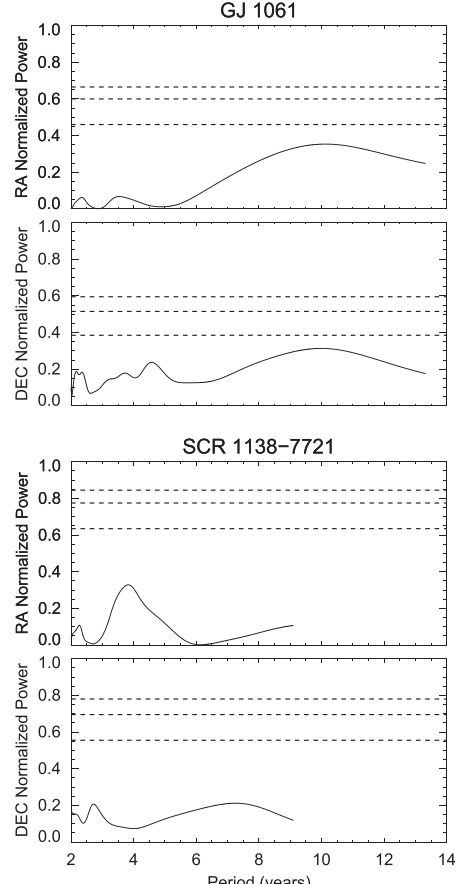

GJ 317

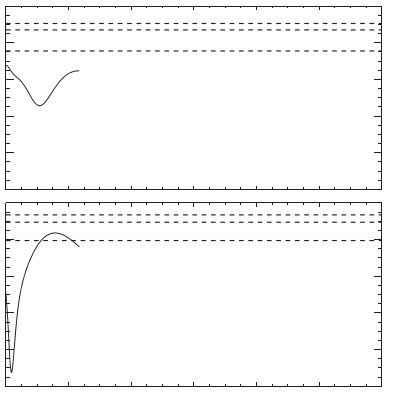

GJ 876
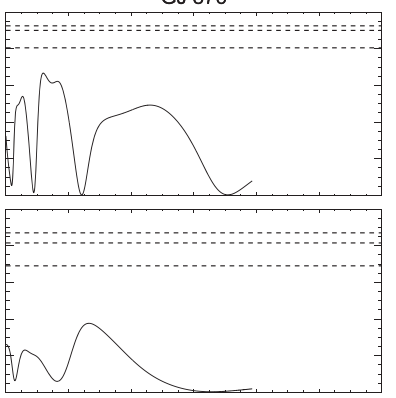

GJ 1128
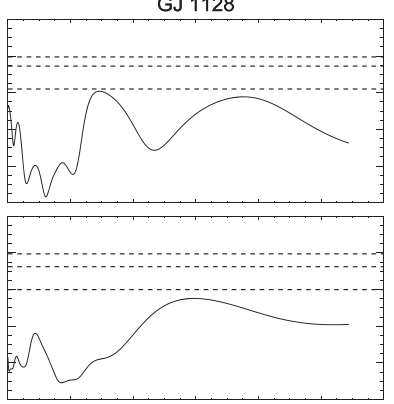

GJ $748 \mathrm{AB}$
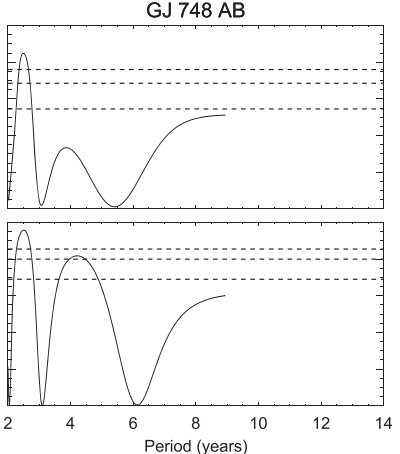

GJ 581

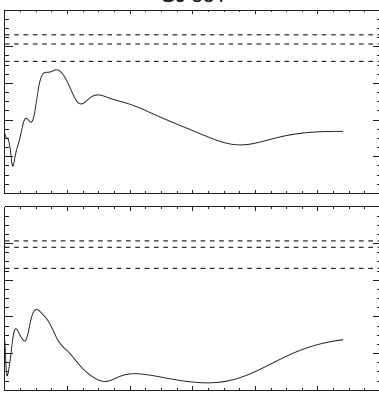

GJ 1214
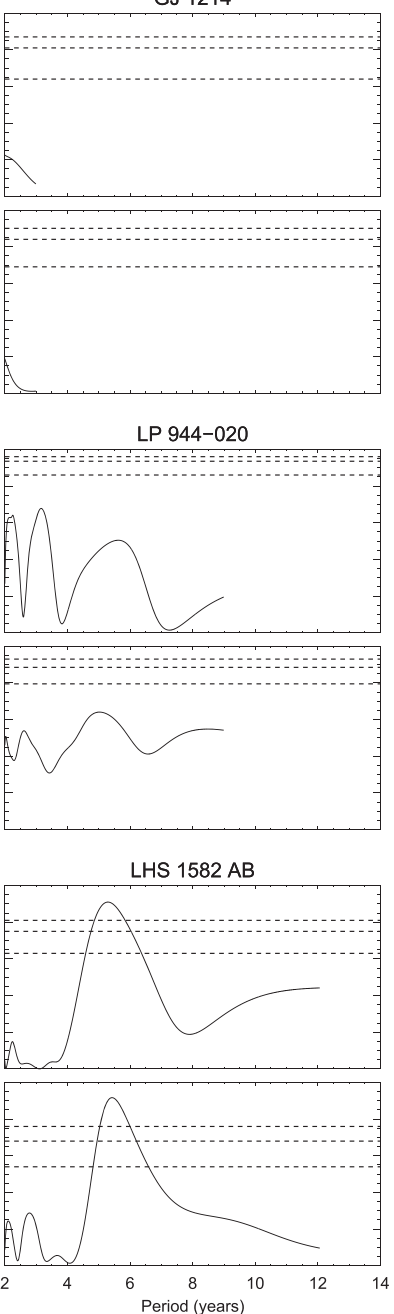

GJ 667 C

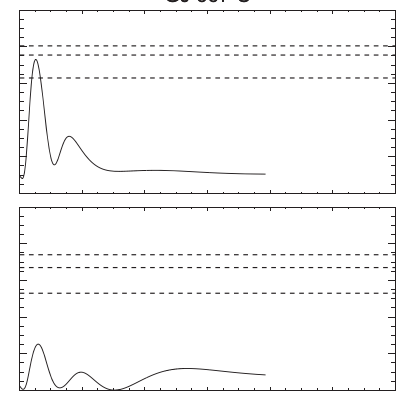

DENIS J1048-3956
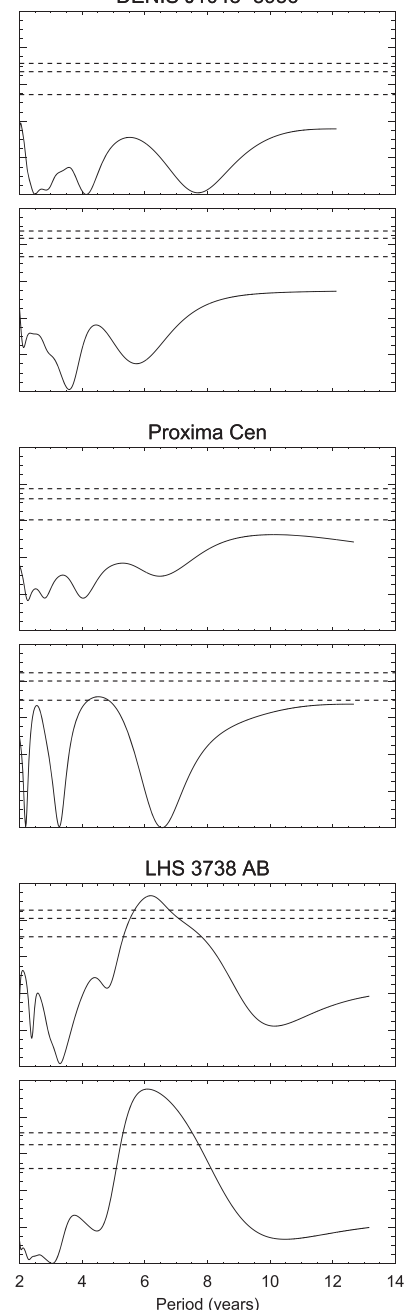

Figure 2. Periodograms and false alarm probabilities (FAPs) for the astrometric residuals. The dashed lines correspond to FAP values of 0.1 (lowest line), 0.01 , and 0.001 (highest line). For the three binaries, the periodograms have significant peaks $(\mathrm{FAP}<0.001)$ at the same period in both R.A. and decl., while none of the other targets have any significant peaks even at the 0.01 level.

on the values plotted in Figure 3, where the lower panel is an inset of the upper. The plotted $\chi_{\text {red }}^{2}$ values were calculated for (1) the stars included in this paper, (2) calibration stars with flat residuals we use to monitor potential fluctuations in equipment and our data reduction pipeline, and (3) additional known binaries with perturbations. We compare these targets' residuals to the case of flat residuals, i.e., an exact astrometric solution with no perturbation. As expected, the binaries with perturbations have large $\chi_{\text {red }}^{2}$ values, indicating that a flat line is a poor fit to the data. The two solid points inside the $\chi_{\text {red }}^{2}=4$ box are long term perturbations with large gaps in the astrometric observations. As more data are collected, those two points will move to larger $\chi_{\text {red }}^{2}$ values outside the box, but we include the points here for completeness. For those stars with no perturbations, all have $\chi_{\text {red }}^{2}$ less than 4 in both axes, and are centered around 1. This indicates that we are accurately calculating our measurement errors, and provides an empirical $\chi_{\text {red }}^{2}$ value of 4 , above which we are sensitive to perturbations.

\section{RESULTS}

Table 1 gives the parallax and proper motion results for the 16 systems, with details about the astrometric observations 

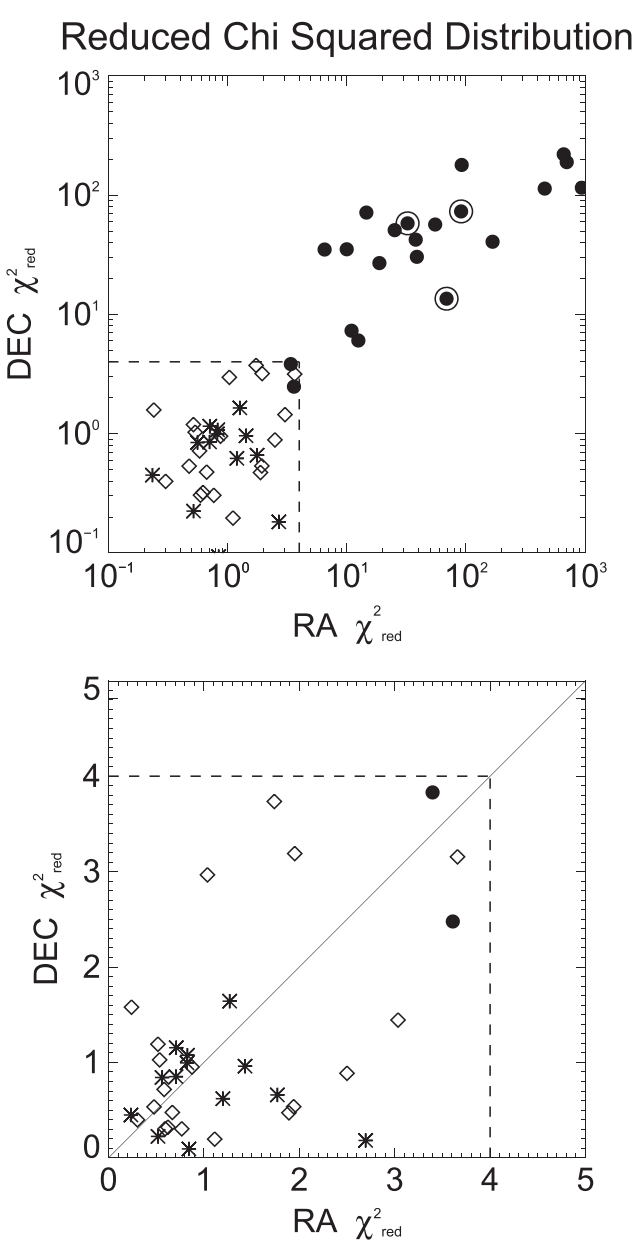

Figure 3. Reduced chi-squared $\left(\chi_{\text {red }}^{2}\right)$ values comparing the astrometric residuals to the case of perfectly flat residuals, i.e., an exact astrometric solution with no perturbation. The lower panel is a zoom of the dotted square region in the upper. Asterisks represent the 13 stars without close stellar secondaries analyzed in this paper. Diamonds represent additional calibration stars with flat residuals. Solid points represent binary stars with perturbations, including the three binaries analyzed in this paper, which are circled. All of the targets with confirmed perturbations have $\chi_{\text {red }}^{2}$ values greater than 4 in both R.A. and decl., with the exception of two stars with long-term perturbations that have gaps in the astrometric observations. All the targets without perturbations have $\chi_{\text {red }}^{2}$ less than 4 in both axes.

(filters used, number of seasons observed, number of frames used in reductions, time coverage, span of time, and the number of reference stars) and results (relative parallaxes, parallax corrections, absolute parallaxes, proper motions, position angles of the proper motions, and the derived tangential velocities based on relative proper motions and parallaxes). All but 2 of the 16 systems have parallax errors of $\sim 2$ mas or less. BD-10 3166 and GJ 876 have larger errors due to combinations of faint reference stars and short exposures. Corrections to absolute parallax are generally less than $\sim 2$ mas, so systematics in the corrections should not significantly affect the results. Three targets (GJ 317, GJ 667C, and BD-10 3166) have corrections of $\sim 3-4$ mas due to reddening of the reference stars, which skews their photometric distance estimates. In these cases, we adopt a generic correction of $1.50 \pm 0.50$ mas. The per observation precision for each target is listed in Column 16, representing the mean of the observation errors in R.A. and decl. The percentage of companions eliminated listed in Column 17 is discussed in Section 5.
Nine of the 16 targets in this paper have parallaxes previously published by RECONS, and are noted in Column 18. The results presented here supersede those published previously by RECONS because additional data and improved reduction techniques have been used, as discussed in detail in Subasavage et al. (2009). The identical parallax of LP 944-020 is also presented in Dieterich et al. (2014) as part of a study of the stellar hydrogen burning limit. For BD-10 3166, we did not run simulations because it is too massive and far away for us to detect any type of substellar companion. However, we do provide the first accurate parallax, and conclude that BD-10 3166 is not physically related to the star with a similar proper motion, LP 731-076, which is 20" away (Bartlett et al. 2014, in preparation).

Photometric and spectroscopic results are provided in Table 2. VRI photometry was taken using the CTIO $0.9 \mathrm{~m}$ (number of nights of photometry in Column 5), with errors in $V R I \lesssim 0.03$ mag (Winters et al. 2011). JHK photometry was retrieved from the Two Micron All Sky Survey (2MASS, Skrutskie et al. 2006) catalog. Spectral types are given in Column 9 with references in Column 10. Mass estimates were calculated as discussed in Section 3. The photometric distances are calculated using the VRIJHK distance relations (number of relations in Column 14) detailed in Henry et al. (2004). For systems with photometric and trigonometric distances that agree within the errors, we conclude that they lack nearly equal luminosity companions. The trigonometric distances of GJ $748 \mathrm{AB}$ and LHS $1582 \mathrm{AB}$ are greater than their photometric distances due to companion contributions to the systems' total flux. The two distances of LHS 3738AB agree well, indicating that the companion is significantly fainter than the primary. GJ 317 and GJ 849 have discrepant (at $3.5 \sigma$ and $3.1 \sigma$, respectively) photometric and trigonometric distances, which does not necessarily mean that these stars have stellar companions, as main sequence stars within the same spectral type can vary somewhat in luminosity.

Bonfils et al. (2013a) note a radial velocity drift in their observations of GJ 849. Montet et al. (2014) also note this drift, and constrain the minimum companion mass to $M \sin i<2.5 M_{\text {Jup }}$. Our astrometry would show a photocenter shift for unequal mass components, as discussed in Section 2. Only components of roughly equal luminosity and mass would provide the additional flux with no perturbation. Such a companion would have been observed to separations as close as $1^{\prime \prime}$ in our images, which corresponds to $\sim 9 \mathrm{AU}$. At a semimajor axis of $9 \mathrm{AU}$, the orbital period is 29.5 years for an equal mass companion. This results in velocities for each component $9.1 \mathrm{~km} \mathrm{~s}^{-1}$ for edge-orbits. Thus, for most orbital inclinations, such a stellar companion is ruled out by the radial velocity data. Therefore, it is unlikely that a stellar companion similar to the primary is contributing to the overluminosity we observe.

Figure 4 shows the range of periods and masses for which $90 \%$ of simulated companions would have been detected, based on the simulations for objects with masses from 0.5 to $80 \mathrm{M}_{\text {Jup }}$. As discussed in Section 3, the masses, periods, and orbital parameters of the simulated companions were assigned randomly from a uniform distribution. For this discussion we set the dividing line between planets and brown dwarfs at 13 $M_{\text {Jup }}$, and the dividing line between brown dwarfs and stars at $80 M_{\text {Jup }}$. The bottom panel of Figure 4 is an inset of the top, showing the best case targets in more detail. The noisy nature 

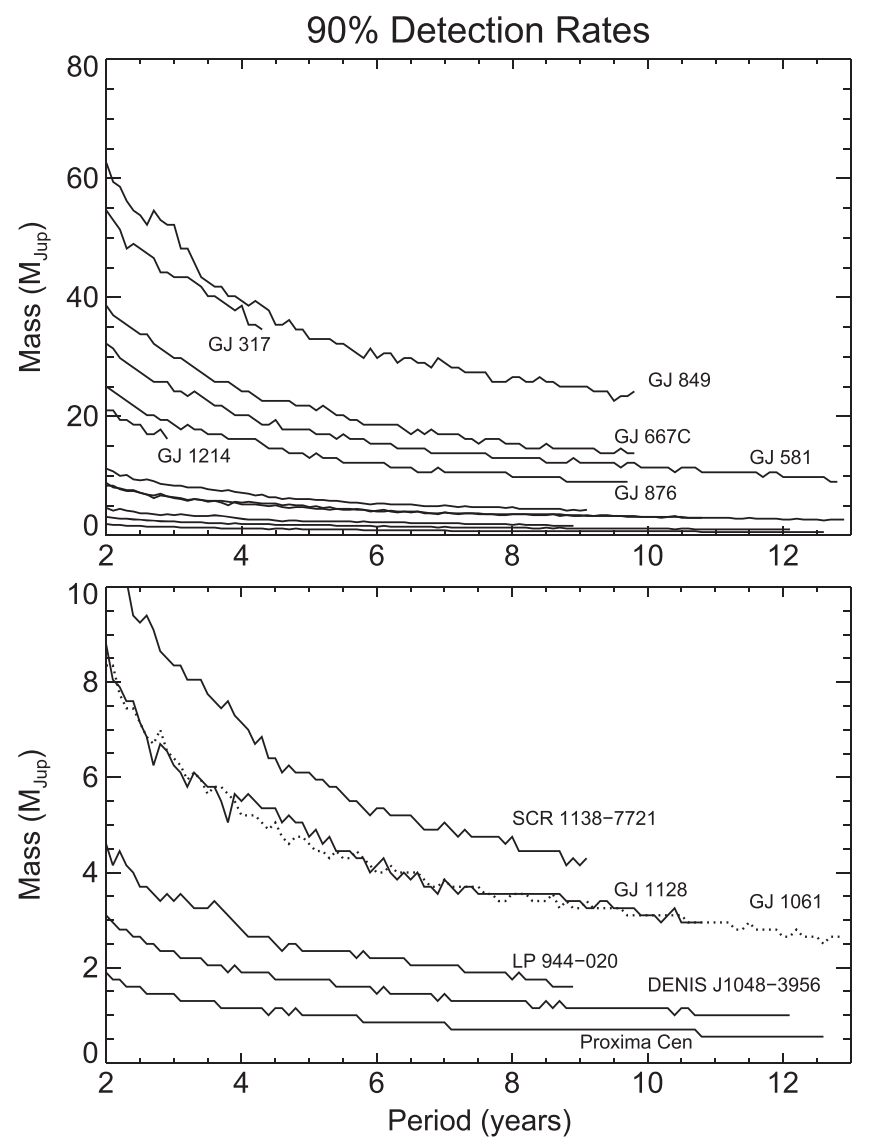

Figure 4. Masses and periods at which $90 \%$ of companions would have been detected, based on simulations of 10 million companions for each star. Companions above the lines would have been detected. The lines end at the length of observations for each target. The six $\mathrm{M}$ dwarfs with known planets are labeled in the top panel. The bottom panel is a zoom of the top, showing in greater detail the six additional $M$ dwarfs that are more favorable to the astrometric detection of planets. GJ 1061 is shown as a dotted line to differentiate it from GJ 1128. Note the different vertical scales, where the top panel represents primarily brown dwarfs and the bottom panel Jovian planets.

of the lines is due to the sizes of the bins used in the simulations. The bin sizes were chosen to achieve a reasonably high resolution, while still having enough simulated companions in each bin. The minimum detectable companion mass is smallest for companions with long periods, which produce the largest amplitude perturbations in the astrometric data. The lower panel indicates that for the best case targets (stars at close distances and of low mass). We are most sensitive to Joviantype planets in Jovian-type orbits.

For a companion at a given mass and orbital period, the amplitude of the resulting astrometric perturbation depends on the orbital parameters of the system, and the mass and distance of the primary. The detection limits we report are based on simulations of companions with a wide range of masses, periods, and orbital parameters. Therefore we give a few representative examples of how the results in Figure 4 translate into astrometric perturbations in mas. In the case of a face-on, circular orbit, a $20 M_{\text {Jup }}$ companion in a 4 year orbit around GJ 581 would cause a 16 mas perturbation, while a $15 M_{\text {Jup }}$ companion in an 8 year orbit would cause a 20 mas perturbation, and a $10 M_{\text {Jup }}$ companion in a 12 year orbit would cause a 17 mas perturbation. For circular, face-on orbits around Proxima Centauri, companions of $1.5,1$, and $0.5 M_{\mathrm{Jup}}$ in orbits of 4,8 , and 12 years would cause perturbations of 12 , 13, and 8 mas, respectively. These values are significantly greater than the per observation precisions listed Table 17.93 mas for GJ 581 and 4.83 mas for Proxima. Thus, the $90 \%$ detection thresholds given in Figure 4 are reasonable

For the four planet hosts observed longer than eight years, Column 17 of Table 1 gives the percentages of simulated brown dwarf companions, ranging from $81 \%-99 \%$, eliminated with orbits between two and eight years, and masses between 13 and $80 M_{\text {Jup }}$. Approximately $92 \%$ of all simulated brown dwarfs have been eliminated as companions to those stars known to host exoplanets. For the six more astrometrically favorable targets, we calculate the percentages of simulated planetary companions eliminated with orbits between two and eight years and masses between 1 and $13 M_{\text {Jup }}$, with results ranging from $69 \%-99 \%$. We have eliminated $\sim 86 \%$ of all simulated planets with masses of 1-13 $M_{\text {Jup }}$ around these six astrometrically favorable stars, and effectively all brown dwarf companions in orbital periods of 2-8 years.

Photocentric orbital solutions for the three binaries are shown in Figure 5 with the corresponding orbital parameters given in Table 3. From our astrometric data for GJ $748 \mathrm{AB}$, we find an orbital period of $2.504 \pm 0.025$ years, which is consistent with the two detailed studies of the system by Franz et al. (1999), who found $P=2.466 \pm 0.008$ years, and Benedict et al. (2001), who found $P=2.469 \pm 0.001$ years using HST Fine Guidance Sensor data. However, we determine an eccentricity of 0.06 , which is inconsistent with the value of 0.45 found in both of the HST studies. We utilized the orbitfitting code described in Hartkopf et al. (1989) and set starting eccentricities of 0.05-0.95 in increments of 0.05; regardless, our data converged to the $e=0.06$ value each time.

The discrepancy between our eccentricity and that of the HST studies is likely due to our observations of GJ $748 \mathrm{AB}$ having been taken at the two different $V$ filters discussed in Section 2.1. While the two filters are photometrically identical within measurable errors (Jao et al. 2011), they are not astrometrically identical. We have analyzed the astrometric residuals for over 500 targets without detectable perturbations in the three different filters $(V, R$, and $I)$ over the length of our observing program. The $R$ and $I$ filters are stable, but astrometric offsets in the $V$ filters are evident over the time period when the problematic "new" $V$ filter was used. These offsets have been mitigated as discussed in Section 2.1, allowing data from both $V$ filters to be used to produce reliable parallax results. In the case of GJ $748 \mathrm{AB}$, we are able to recover the correct period, but the offsets in the residuals are likely contributing to the errant eccentricity. We presently do not possess enough observations to perform a reduction of GJ $748 \mathrm{AB}$ without the problematic $V$ filter data. We include the current solution because it is our only system for which an accurate period has been published, against which to compare our results.

In contrast to our photocenter data, the FGS observations resolve the system into two components at 15 epochs over 1.8 years. They are exquisitely sensitive to the separation and position angle of the secondary from the primary, and are to be preferred to our ground-based results for the eccentricity. A clever suggestion by Hugh Harris of USNO has been suggested to solve this dilemma. Because in an unresolved system the center of mass location is unknown, the zero points for the residuals in R.A. and decl. are unknown. By shifting the zero 

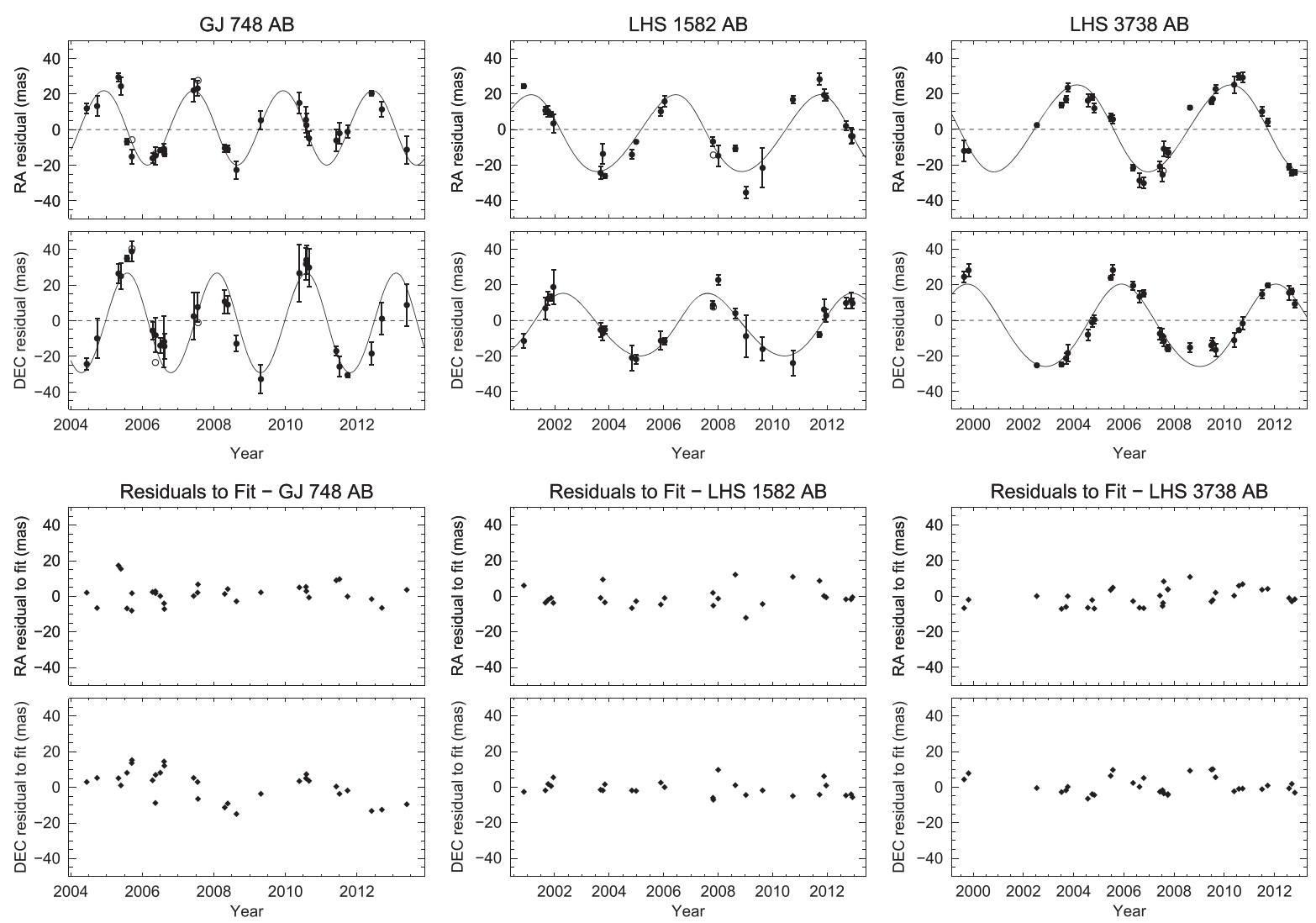

Figure 5. Top panels show the orbital fits for the astrometric residuals of three confirmed binaries. Filled circles represent the mean of typically three to five frames taken in a single night. Open circles represent nights for which there is only one frame and are not used to derive the fit. Bottom panels show the residuals to the orbital fits. All panels are on a \pm 50 mas scale.

Table 3

Orbital Parameters of Known Binaries

\begin{tabular}{|c|c|c|c|c|c|c|c|}
\hline Name & $\begin{array}{c}P \\
\text { (years) }\end{array}$ & $T_{0}$ & $\begin{array}{l}a \text { phot } \\
\text { (mas) }\end{array}$ & $e$ & $\begin{array}{c}i \\
(\operatorname{deg})\end{array}$ & $\begin{array}{l}\text { Long. Peri. }(\omega) \\
\quad(\mathrm{deg})\end{array}$ & $\begin{array}{c}\text { Long. Node }(\Omega) \\
(\operatorname{deg})\end{array}$ \\
\hline (1) & $(2)$ & (3) & (4) & $(5)$ & $(6)$ & $(7)$ & $(8)$ \\
\hline GJ 748AB & $2.504 \pm 0.025$ & $2005.86 \pm 0.25$ & $28.1 \pm 1.6$ & $0.06 \pm 0.04$ & $137.8 \pm 9.0$ & $218.2 \pm 40.3$ & $173.6 \pm 12.1$ \\
\hline LHS $1582 \mathrm{AB}$ & $5.309 \pm 0.049$ & $2001.84 \pm 0.14$ & $21.9 \pm 1.3$ & $0.17 \pm 0.03$ & $143.6 \pm 7.5$ & $62.0 \pm 14.3$ & $97.9 \pm 12.4$ \\
\hline LHS $3738 \mathrm{AB}$ & $6.141 \pm 0.059$ & $2005.73 \pm 0.16$ & $28.1 \pm 1.2$ & $0.12 \pm 0.02$ & $131.8 \pm 4.1$ & $130.5 \pm 11.2$ & $130.5 \pm 5.1$ \\
\hline
\end{tabular}

points and fitting the residuals, a different eccentricity may be derived. We await the acquisition of resolved data for several more systems before exploring this technique so that a robust analysis can be accomplished. At present errors on the eccentricities in Table 3 should be treated with caution. The orbital solutions for LHS 1582AB and LHS 3738AB are updated and improved over those presented in Riedel et al. (2010), which were the first orbits presented for each system. In addition to demonstrating the astrometric detection and characterization of unresolved companions, these results can provide additional dynamically determined masses for $\mathbf{M}$ dwarfs, once the systems have been resolved.

\section{DISCUSSION}

In narrow-angle field astrometry programs such as the one outlined here, the lowest mass companions detectable by astrometry are dependent on a number of factors. These include the apparent brightness of the host star and availability of suitable reference stars, which affect the precision of the astrometric measurements. Additionally, the target star mass, companion mass, the luminosity ratio, orbital separation, and system distance determine the size of the astrometric perturbation. The M dwarfs with planets that have been discovered so far, including the six in this paper, are not the most favorable to our astrometric observations. They are among the brighter red dwarfs, with the latest type stars at $\mathrm{M} 3.5 \mathrm{~V}$ for the radial velocity detections included in this paper. We are rarely sensitive to planets around these stars. However, we are able to rule out the presence of a large fraction of potential brown dwarf companions with masses of $13-80 M_{\text {Jup }}$. As observations continue, we will be able to rule out companions at longer periods, particularly in the cases of GJ 317 and GJ 1214, which we have so far only observed for 3-4 years. In addition, we have demonstrated that we are sensitive to planets with masses of $1-13 M_{\mathrm{Jup}}$ around $\mathrm{M}$ dwarfs that are more favorable to astrometric observations. Of particular interest, we find that Proxima Centauri has no companions more massive than $2 M_{\text {Jup }}$ with periods of 2-5 years and more massive than $1 M_{\text {Jup }}$ for $5-12$ years. This is to be contrasted to the results of other 
Table 4

Planets Orbiting M Dwarfs within 25 pc

\begin{tabular}{|c|c|c|c|c|c|c|c|c|c|}
\hline (1) & (2) & (3) & $\begin{array}{c}\pi_{\text {mean }} \\
(\text { mas }) \\
(4)\end{array}$ & $\begin{array}{l}\mathrm{N}_{\pi} \\
(5)\end{array}$ & (6) & $\begin{array}{c}\mathrm{M} \sin (i) \\
\left(\mathrm{M}_{\text {Jup }}\right) \\
(7)\end{array}$ & $\begin{array}{c}\mathrm{P} \\
\text { (years) } \\
(8)\end{array}$ & $\begin{array}{l}\text { Ref. } \\
\text { (9) }\end{array}$ & (10) \\
\hline GJ $163 \mathrm{c}$ & & & & & & 0.02 & 0.070 & 9 & \\
\hline GJ $163 \mathrm{~d}$ & & & & & & 0.09 & 1.654 & 9 & \\
\hline GJ 176 b & 044255.7 & +185729 & $110.00 \pm 2.00$ & 2 & 1,2 & 0.03 & 0.024 & 10 & \\
\hline LHS $2335 \mathrm{~b}$ & 105835.0 & -310838 & $50.55 \pm 1.55$ & 1 & 5 & 0.02 & 0.007 & 12 & \\
\hline GJ 433 b & 113526.9 & $\begin{array}{lll}-32 & 32 & 23\end{array}$ & $112.09 \pm 1.43$ & 2 & 1,2 & 0.02 & 0.020 & 13 & $\mathrm{a}$ \\
\hline GJ 1148 b & 114144.6 & +424507 & $88.81 \pm 2.14$ & 2 & 1,2 & 0.30 & 0.113 & 14 & \\
\hline GJ 436 b & 114211.0 & +264223 & $98.95 \pm 2.07$ & 2 & 1,2 & 0.07 & 0.007 & 15 & \\
\hline GJ 581 b & 151926.0 & -074320 & $159.28 \pm 1.32$ & 3 & $1,2,3$ & 0.05 & 0.015 & 16 & \\
\hline GJ $581 \mathrm{c}$ & & & & & & 0.02 & 0.035 & 16 & \\
\hline GJ $581 \mathrm{~d}$ & & & & & & 0.02 & 0.183 & 16 & \\
\hline GJ $667 \mathrm{C} \mathrm{b}$ & 171857.1 & -345923 & $138.24 \pm 0.57$ & 3 & $1,3,7$ & 0.02 & 0.020 & 13 & $\mathrm{a}$ \\
\hline GJ $667 \mathrm{C} \mathrm{c}$ & & & & & & 0.01 & 0.077 & 13 & \\
\hline GJ 674 b & 172839.9 & -465342 & $220.11 \pm 1.39$ & 2 & 1,2 & 0.03 & 0.013 & 20 & \\
\hline GJ 832 b & 213333.9 & -490032 & $202.03 \pm 1.00$ & 2 & 1,2 & 0.64 & 9.353 & 21 & \\
\hline GJ 849 b & 220940.3 & -043826 & $113.46 \pm 1.32$ & 3 & $1,2,3$ & 0.90 & 5.241 & 22 & $\mathrm{a}$ \\
\hline GJ 876 b & 225316.7 & -141549 & $214.45 \pm 0.57$ & 4 & $1,2,3,8$ & 1.95 & 0.167 & 23 & \\
\hline GJ $876 \mathrm{c}$ & & & & & & 0.61 & 0.082 & 23 & \\
\hline GJ $876 \mathrm{~d}$ & & & & & & 0.02 & 0.005 & 23 & \\
\hline GJ 876 e & & & & & & 0.04 & 0.342 & 23 & \\
\hline
\end{tabular}

Notes. To provide a uniform format, minimum masses and orbital periods are in some cases rounded to fewer significant digits than in the original publications. (a) Additional companion(s) not listed on both exoplanet.eu and exoplanets.org; (b) transiting planet; all others are radial velocity detections. Recent discoveries GJ 191 b/c (Anglada-Escudé et al. 2014) and GJ 687 b (Burt et al. 2014) were not listed on exoplanets.org as of 2014 July 1.

References. (1) Yale Parallax Catalog, van Altena et al. (1995); (2) Hipparcos, van Leeuwen (2007); (3) This work; (4) Anglada-Escudé et al. (2012); (5) Riedel et al. (2010); (6) Anglada-Escudé et al. (2013): (7) Fabricius \& Makarov (2000): (8) Benedict et al. (2002); (9) Bonfils et al. (2013); (10) Forveille et al. (2009); (11) Howard et al. (2010); (12) Bonfils et al. (2011); (13) Delfosse et al. (2013); (14) Haghighipour et al. (2010); (15) Maness et al. (2007); (16) Mayor et al. (2009); (17) Apps et al. (2010); (18) Johnson et al. (2010); (19) Harpsøe et al. (2013); (20) Bonfils et al. (2007); (21) Bailey et al. (2009); (22) Montet et al. (2014); (23) Rivera et al. (2010).

companion searches of Proxima Centauri. Using HST Fine Guidance Sensor data, Benedict et al. (1999) report a companion detection sensitivity of $1 M_{\text {Jup }}$ at a 60 day period. Based on 7 years of RV observations, Endl \& Kürster (2008) preclude the presence of companions with $M \sin (i) \geqslant 1 M_{\text {Nep }}\left(0.05 M_{\text {Jup }}\right)$ at periods $\leqslant 2.7$ years. Together these studies eliminate all Jupiter mass planets around Proxima Centauri for orbital periods out to 12 years.

In the broader context, these results are consistent with recently published searches for Jovian companions to M dwarfs at shorter orbital periods. Transit searches are unlikely to detect companions at Jovian orbits, due to the narrow range of detectable inclinations. Berta et al. (2013) found no Jupitersized planets in their transit search, which is most sensitive to companions at orbital periods less than 10 days, and conclude that such planets rarely orbit M dwarfs. Based on the first two years of their astrometric search, Sahlmann et al. (2014) find no planetary mass companions to the $20 \mathrm{M}$ and L dwarfs they observed. They determine the occurrence rate of planets more massive than $\sim 5 M_{\text {Jup }}$ to have an upper limit of $9 \%$.

The longer time coverage of the astrometric results presented in this work overlap most closely with radial velocity results.
Bonfils et al. (2013a) report detection limits based on radial velocity measurements for 102 nearby $\mathrm{M}$ dwarfs, of which six are featured in this paper. Among the $102 \mathrm{M}$ dwarfs searched, they confirm only two planets with orbital periods longer than 100 days. At an orbital period of 1000 days (2.7 years), they report a detection sensitivity of $M \sin (i) \leqslant 2 M_{\text {Jup }}$ around $90 \%$ of stars observed, and $M \sin (i) \leqslant 30 M_{\text {Jup }}$ at 10,000 days (27 years). Montet et al. (2014) report that $6.5 \% \pm 3.0 \%$ of $\mathrm{M}$ dwarfs host a 1 to $13 M_{\text {Jup }}$ planet at a separation less than $20 \mathrm{AU}$, based on their radial velocity survey and high resolution imaging.

The results presented here are among the first astrometric searches for Jovian companions at Jovian orbits, and fill in relatively unexplored mass and period parameter spacemodels of how $\mathrm{M}$ dwarf planetary systems form and evolve must now explain the lack of massive companions with long period orbits like those in our Solar System. Looking forward, the Gaia mission may detect up to 2600 planets within $100 \mathrm{pc}$ (Sozzetti et al. 2014). However, its ability to determine accurate masses and orbits will be limited to orbital periods less than 6 years, a fraction of the time coverage of our groundbased astrometric observations. 
Finding nearby $\mathrm{M}$ dwarfs with planets remains an important challenge, as the closest planets are the brightest and most easily studied, and $\mathrm{M}$ dwarfs dominate the stellar population. As part of its mission to characterize the solar neighborhood, RECONS is developing a database of all objects with accurate trigonometric parallaxes placing them within $25 \mathrm{pc}\left(\pi_{\text {trig }} \geqslant 40\right.$ mas with an error $\leqslant 10$ mas). For an extrasolar planet to be included in the RECONS Database it must orbit a star that meets the above criteria, or be a free-floating object with a comparable parallax, and be listed in both the Extrasolar Planets Encyclopaedia (exoplanet.eu) and the Exoplanet Orbit Database exoplanets.org, Wright et al. 2011). The RECONS Database currently contains 1074 systems having $\mathrm{M}$ dwarf primaries $\left(9.0 \leqslant M_{V} \leqslant 21.0\right)$ within $25 \mathrm{pc}$, only 17 of which have detected exoplanets as of 2014 July 1, listed in Table 4. The error-weighted mean parallax for each system is given in Column 4, including the parallaxes in this work and published values. Minimum masses and orbital periods for planets with references are listed in Columns 7-9. Note that only 3 of the 26 reported planets have masses greater than Jupiter's. Based on the $36 \mathrm{M}$ dwarf primaries within $5 \mathrm{pc}$ (Henry 2013), we anticipate that there are $4500 \mathrm{M}$ dwarf primaries within 25 parsecs, yet only 17 so far have been found to host planets. Clearly, many planets lurk undetected in the solar neighborhood. Discovering these planets will require a wide variety of survey techniques, and as sensitivities are improved, astrometry will continue to play an important role.

We wish to thank Cassy Davison for her assistance developing the simulations, and Sergio Dieterich for helpful conversations regarding brown dwarf luminosities, and his assistance in surveying the existing literature. We also wish to thank Hugh Harris for his helpful suggestion regarding the eccentricities of unresolved binaries. This effort has been supported by the National Science Foundation via grants AST 05-07711 and AST 09-08402, and the long-term cooperative efforts of the National Optical Astronomy Observatories and the members of the SMARTS Consortium. This research has made use of the Exoplanet Orbit Database and the Exoplanet Data Explorer at exoplanets.org, as well as the Extrasolar Planet Encyclopaedia at exoplanet.eu. This research has also made use of Aladin and the SIMBAD database, operated at CDS, Strasbourg, France.

Facilities: CTIO:0.9m, CTIO:1.5m, CTIO:2MASS, FLWO:2MASS

\section{REFERENCES}

Anglada-Escudé, G., Arriagada, P., Tuomi, M., et al. 2014, MNRAS, 443, L89 Anglada-Escudé, G., Boss, A. P., Weinberger, A. J., et al. 2012, ApJ, 746, 37 Anglada-Escudé, G., Rojas-Ayala, B., Boss, A. P., Weinberger, A. J., \& Lloyd, J. P. 2013, A\&A, 551, A48

Apps, K., Clubb, K. I., Fischer, D. A., et al. 2010, PASP, 122, 156
Bailey, J., Butler, R. P., Tinney, C. G., et al. 2009, ApJ, 690, 743 Benedict, G. F., McArthur, B., Chappell, D. W., et al. 1999, AJ, 118, 1086 Benedict, G. F., McArthur, B. E., Franz, O. G., et al. 2001, AJ, 121, 1607 Benedict, G. F., McArthur, B. E., Forveille, T., et al. 2002, ApJ, 581, L115 Berta, Z. K., Irwin, J., \& Charbonneau, D. 2013, ApJ, 775, 91 Bertin, E., \& Arnouts, S. 1996, A\&AS, 117, 393

Bonfils, X., Mayor, M., Delfosse, X., et al. 2007, A\&A, 474, 293

Bonfils, X., Gillon, M., Forveille, T., et al. 2011, A\&A, 528, A111

Bonfils, X., Delfosse, X., Udry, S., et al. 2013, A\&A, 549, A109

Bonfils, X., Lo Curto, G., Correia, A. C. M., et al. 2013, A\&A, 556, A110

Burt, J., Vogt, S. S., Butler, R. P., et al. 2014, ApJ, 789, 114

Delfosse, X., Bonfils, X., Forveille, T., et al. 2013, A\&A, 553, A8

Dieterich, S. B., Henry, T. J., Jao, W.-C., et al. 2014, AJ, 147, 94

Dressing, C. D., \& Charbonneau, D. 2013, ApJ, 767, 95

Endl, M., \& Kürster, M. 2008, A\&A, 488, 1149

Fabricius, C., \& Makarov, V. V. 2000, A\&AS, 144, 45

Forveille, T., Bonfils, X., Delfosse, X., et al. 2009, A\&A, 493, 645

Franz, O. G., Wasserman, L. H., Benedict, G. F., et al. 1999, in ASP Conf. Ser. 194, Working on the Fringe: Optical and IR Interferometry from Ground and Space, ed. S. Unwin, \& R. Stachnik (San Francisco, CA: ASP), 28

Frescura, F. A. M., Engelbrecht, C. A., \& Frank, B. S. 2008, MNRAS, 388,1693

Graham, J. A. 1982, PASP, 94, 244

Haghighipour, N., Vogt, S. S., Butler, R. P., et al. 2010, ApJ, 715, 271

Harpsøe, K. B. W., Hardis, S., Hinse, T. C., et al. 2013, A\&A, 549, A10

Hartkopf, W. I., McAlister, H. A., \& Franz, O. G. 1989, AJ, 98, 1014

Hawley, S. L., Gizis, J. E., \& Reid, I. N. 1996, AJ, 112, 2799

Henry, T. J., \& McCarthy, D. W. Jr. 1993, AJ, 106, 773

Henry, T. J., Ianna, P. A., Kirkpatrick, J. D., \& Jahreiß, H. 1997, AJ, 114, 388

Henry, T. J., Franz, O. G., Wasserman, L. H., et al. 1999, ApJ, 512, 864

Henry, T. J., Subasavage, J. P., Brown, M. A., et al. 2004, AJ, 128, 2460

Henry, T. J., Jao, W.-C., Subasavage, J. P., et al. 2006, AJ, 132, 2360

Henry, T. J. 2013, in Royal Astronomical Society of Canada Observer's Handbook 2013, ed. D. M. F. Chapman (Toronto: Royal Astronomical Society of Canada)

Howard, A. W., Johnson, J. A., Marcy, G. W., et al. 2010, ApJ, 721, 1467

Jao, W.-C., Henry, T. J., Subasavage, J. P., et al. 2003, AJ, 125, 332

Jao, W.-C., Henry, T. J., Subasavage, J. P., et al. 2005, AJ, 129, 1954

Jao, W.-C., Henry, T. J., Subasavage, J. P., et al. 2011, AJ, 141, 117

Johnson, J. A., Howard, A. W., Marcy, G. W., et al. 2010, PASP, 122, 149

Landolt, A. U. 1992, AJ, 104, 340

Landolt, A. U. 2007, AJ, 133, 2502

Maness, H. L., Marcy, G. W., Ford, E. B., et al. 2007, PASP, 119, 90

Mayor, M., Bonfils, X., Forveille, T., et al. 2009, A\&A, 507, 487

Montet, B. T., Crepp, J. R., Johnson, J. A., Howard, A. W., \& Marcy, G. W. 2014, ApJ, 781, 2

Reid, I. N., Hawley, S. L., \& Gizis, J. E. 1995, AJ, 110, 1838

Riedel, A. R., Subasavage, J. P., Finch, C. T., et al. 2010, AJ, 140, 897

Rivera, E. J., Laughlin, G., Butler, R. P., et al. 2010, ApJ, 719, 890

Robertson, P., Mahadevan, S., Endl, M., \& Roy, A. 2014, Sci, 345, 440

Sahlmann, J., Lazorenko, P. F., Ségransan, D., et al. 2014, A\&A, 565, A20

Skrutskie, M. F., Cutri, R. M., Stiening, R., et al. 2006, AJ, 131, 1163

Sozzetti, A., Giacobbe, P., Lattanzi, M. G., et al. 2014, MNRAS, 437, 497

Subasavage, J. P., Jao, W.-C., Henry, T. J., et al. 2009, AJ, 137, 4547

van Altena, W. F., Lee, J. T., \& Hoffleit, D. 1995, in The General Catalogue of Trigonometric Stellar Parallaxes (4th ed.; New Haven, CT: Yale Univ. Obs.)

van de Kamp, P. 1967, in Principles of Astrometry (San Francisco: W.H. Freeman \& company)

van Leeuwen, F. 2007, A\&A, 474, 653

Winters, J. G., Henry, T. J., Jao, W.-C., et al. 2011, AJ, 141, 21

Wright, J. T., Fakhouri, O., Marcy, G. W., et al. 2011, PASP, 123, 412

Zechmeister, M., \& Kürster, M. 2009, A\&A, 496, 577 